\title{
Multimode vibration suppression with passive two-terminal distributed network incorporating piezoceramic transducers
}

\author{
R.C. Batra ${ }^{\mathrm{a}, *}$, F. dell'Isola ${ }^{\mathrm{b}}$, S. Vidoli $^{\mathrm{b}}$, D. Vigilante $^{\mathrm{a}}$ \\ a Department of Engineering Science and Mechanics, MC 0219, Virginia Polytechnic Institute and State University, \\ Blacksburg, VA 24061, USA \\ b Dipartimento di Ingegneria Strutturale e Geotecnica, Università degli studi di Roma "La Sapienza", \\ Via Eudossiana 18, 00184 Rome, Italy
}

Received 19 October 2004

\begin{abstract}
The design of an inductance/capacitance two-terminal distributed passive electric network for simultaneously controlling several flexural modes of vibration is discussed. The efficiency of the network to annul multimode vibrations of simply supported, clamped-clamped and cantilever beams is demonstrated.

(C) 2004 Elsevier Ltd. All rights reserved.
\end{abstract}

Keywords: Self-controlled beams; Simultaneous control of several vibration modes; Electromechanical coupling

\section{Introduction}

The number of industrial applications of smart structures that can self control their vibrations has been steadily growing over the last several years. The traditional design has involved embedding sensors and actuators into the system, the former to sense and the latter to control the motion (following the ideas described in Hagood and von Flotow (1991)). It is now possible to design an integrated system in which the control subsystem is not based on the paradigm of sensing-evaluating-actuating, but is an integral part of the system. Thus the structure is self-controlled. Examples of such systems are the piezoelectromechanical (PEM) beams and plates studied in Alves Rade and Valder (2000), dell'Isola and Vidoli (1998), Lesieutre (1998), Vidoli and dell'Isola (2000), Vidoli and dell'Isola (2001), Hagood and von Flotow (1991) and Park

\footnotetext{
Corresponding author. Tel: +1 5402316051; fax: +1 5402314574.

E-mail addresses: rbatra@vt.edu (R.C. Batra), francesco.dellisola@uniroma1.it (F. dell'Isola), stefano.vidoli@uniroma1.it (S. Vidoli), dvigilan@vt.edu (D. Vigilante).
} 
and Inman (2003). The design of such systems involves the transfer, by means of a distributed array of piezoelectric transducers, of the mechanical energy into the electric energy which is then dissipated. The electric circuit does not include an active controller and, therefore, no external "intelligence" is required to damp out mechanical vibrations.

We study here the problem of controlling flexural vibrations of a beam. More specifically, we design the two-terminal network forming the basic modules of a periodic transmission line that can simultaneously control several flexural modes of vibration of a beam. We generalize the results found in Fleming and Reza Moheimani (2002), Wu (1998) and Hollkamp (1994) where the multifrequency control concept is exploited for systems in which a single PZT actuator feeds a multiresonant electric circuit.

The paper is organized as follows. Section 2 reviews elementary results for vibrations of uncoupled mechanical and electrical systems and gives a mathematical formulation of the problem. Section 3 describes the design of an electrical network for simultaneously controlling a given number of flexural modes of vibration of a beam. Applications of the proposed device to annul vibrations of a simply supported beam, clamped-clamped beam, and a cantilever beam are described in Section 4. Section 5 summarizes conclusions. Three appendices give detailed derivation of some of the results presented in the paper. The derivation of governing equations of the electric network is given in Appendix A. In Appendix B, we describe the procedure used to numerically solve equations governing the coupled electromechanical deformations of a beam with PZT patches. The impedance required in the electrical circuit is determined in Appendix C.

\section{Statement of the problem}

\subsection{Mathematical framework}

Consider two systems governed by the following partial differential equations and boundary conditions:

$$
\begin{aligned}
& L_{\mathrm{m}}(w)+D_{\mathrm{m}}(w)=0, \quad D_{\mathrm{e}}^{1}\left(L_{\mathrm{e}}(v)\right)+D_{\mathrm{e}}^{2}(v)=0, \\
& B_{\mathrm{m}}(w)=0, \quad B_{\mathrm{e}}(v)=0 .
\end{aligned}
$$

Here $w$ and $v$ are kinematical descriptors, $L_{\mathrm{m}}$ and $L_{\mathrm{e}}$ are linear self-adjoint spatial differential operators, and $D_{\mathrm{m}}, D_{\mathrm{e}}^{1}$ and $D_{\mathrm{e}}^{2}$ are linear differential operators in the time domain. Eq. (2) express the pertinent boundary conditions. The subscripts " $\mathrm{m}$ " and "e" stand for mechanical and electrical systems respectively.

We assume that $L_{\mathrm{m}}, D_{\mathrm{m}}, B_{\mathrm{m}}, L_{\mathrm{e}}$ and $B_{\mathrm{e}}$ are given; moreover $D_{\mathrm{m}}$ is assumed to be a second order time operator representing the standard dynamics. In order to establish a multiresonance phenomenon between the $m$ and the $e$-subsystems, electric circuits are designed in Vidoli and dell'Isola (2000) and Andreaus et al. (2004) such that

$$
L_{\mathrm{e}}=L_{\mathrm{m}}, \quad D_{\mathrm{e}}^{1}=\text { Identity }, \quad D_{\mathrm{e}}^{2}=D_{\mathrm{m}} .
$$

In other words the goal of the work presented in Vidoli and dell'Isola (2000) and Andreaus et al. (2004) was to find the circuit topology so that the circuit had the same spatial differential operator as the mechanical system to be controlled. Instead here we assume $L_{\mathrm{e}}$ to be given (the simplest connection is chosen) and find the time differential operators $D_{\mathrm{e}}^{1}$ and $D_{\mathrm{e}}^{2}$. More precisely, $D_{\mathrm{e}}^{1}$ and $D_{\mathrm{e}}^{2}$ are chosen so that $n$ eigenfrequencies of the boundary-value problem defined by Eqs. $(1)_{1}$ and $(2)_{1}$ coincide with the $n$ eigenfrequencies of the boundary-value problem specified by Eqs. $(1)_{2}$ and $(2)_{2}$. This reduces to tuning the $e$-system on a certain number of frequencies of the $m$-system thus establishing a multiresonance phenomenon.

To achieve this goal, we seek standing wave solutions of Eqs. (1) and (2)

$$
\{w, v\}^{\mathrm{T}}=\{W, V\}^{\mathrm{T}} \mathrm{e}^{\mathrm{i} k \xi} \mathrm{e}^{p t},
$$


where $k$ is the wavelength, $p$ the frequency, $\mathrm{i}=\sqrt{-1}$, and $W$ and $V$ are amplitudes. Substitution for $w$ and $v$ from (3) into (1) and (2) gives

$$
\begin{aligned}
& S_{\mathrm{m}}(k)-T_{\mathrm{m}}(p)=0, \quad S_{\mathrm{e}}(k)-F(p)=0, \\
& P_{\mathrm{m}}(k)=0, \quad P_{\mathrm{e}}(k)=0,
\end{aligned}
$$

where

$$
F(p)=T_{\mathrm{e}}^{2}(p) / T_{\mathrm{e}}^{1}(p),
$$

and $S_{\mathrm{m}}, T_{\mathrm{m}}, T_{\mathrm{e}}^{1}$ and $T_{\mathrm{e}}^{2}$ are polynomial functions, and $P_{\mathrm{m}}$ and $P_{\mathrm{e}}$ are polynomial combinations of exponential functions constraining the admissible values for the wavelengths $k$. Indeed boundary conditions $(4)_{3,4}$ constrain the admissible discrete values of $\alpha_{j}$ and $\beta_{j}, j=1,2, \ldots$, i.e., the $m$-wavelengths and the $e$-wavelengths respectively. As usual, once the admissible wavelengths have been found, eigenfrequencies $p$ are solutions of Eqs. (4) $)_{1,2}$. Let

$$
s_{j}=\left\{p: S_{\mathrm{m}}\left(\alpha_{j}\right)-T_{\mathrm{m}}(p)=0\right\}, \quad \sigma_{j}=\left\{p: S_{\mathrm{e}}\left(\beta_{j}\right)-F(p)=0\right\} .
$$

For each $\alpha_{j}$ (i.e., for each mode shape), the set $s_{j}$ is a couple of complex conjugate solutions, since, by the assumptions on $D_{\mathrm{m}}, T_{\mathrm{m}}$ is a second order polynomial with real coefficients. However, for each $\beta_{j}$, the set $\sigma_{j}$ can be a larger set of complex conjugate solutions since $F$ is a rational function with real coefficients.

Therefore we need to solve the following

Problem 1. Find the rational function $F(p)$ so that, for given sets $\left(\alpha_{1}, \alpha_{2}, \ldots, \alpha_{n}\right)$ and $\left(\beta_{1}, \beta_{2}, \ldots, \beta_{n}\right)$ of $m$ wavelengths and e-wavelengths respectively,

$$
s_{j} \subset \sigma_{j}, \quad j=1,2, \ldots, n .
$$

Hence, once the two sets of $n$ corresponding mode shapes have been chosen, $n$ self-resonance phenomena are established between them.

\subsection{Description of the system}

The aforementioned tuning procedure is illustrated by a suitable choice of the $m$ - and the $e$-systems, namely an Euler beam and an electric transmission line respectively.

Fig. 1 shows a sketch of a typical problem studied: the system is comprised of a prismatic rectangular beam with a set of rectangular piezoceramic (PZT) actuators affixed to its top and/or bottom surfaces. The abbreviation PZT is used for a generic piezoelectric ceramic rather than a specific material. The

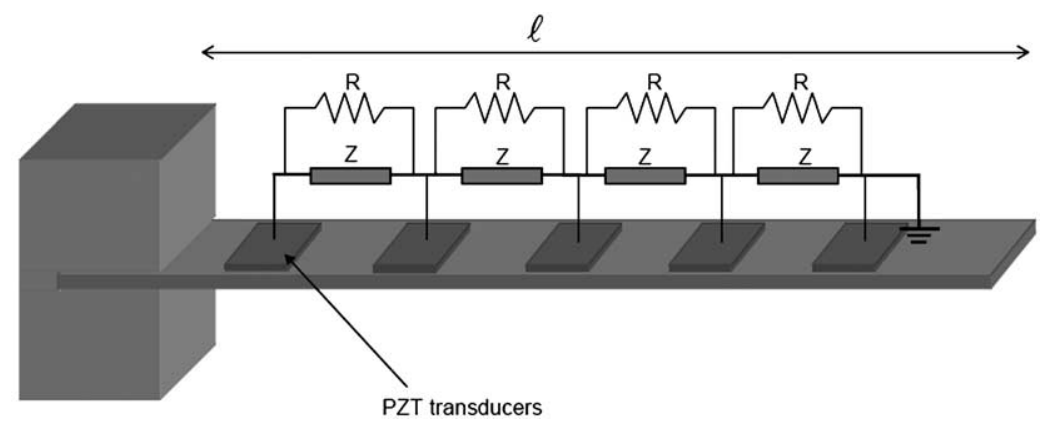

Fig. 1. Sketch of a typical problem studied. $Z$ is the impedance of a LC two-terminal network, and $R$ is a resistor. 
PZT patches are interconnected through resistors $R$ and electric impedances $Z$ to form an inductorcapacitor (LC) two-terminal network (TTN).

Our goal is to determine the impedance $Z$ so that some frequencies of the electrical network (the $e$ system) match with the desired frequencies of the beam (the $m$-system). The transfer of mechanical energy into electrical energy is assured by the electromechanical coupling of the PZTs distributed along the beam. Even though a finite number of PZT actuators are employed, we treat the electric circuit as a continuum. This is reasonable since the wavelengths of the mechanical disturbances to be controlled are much larger than the distance between adjoining PZT patches.

\subsection{1. m-system}

The beam is modeled as an Euler beam; hence, with a suitable choice of non-dimensional variables, its equation of motion can be written as

$$
w^{i v}+\ddot{w}=0,
$$

where $w$ is the dimensionless transverse displacement, a prime (superimposed dot) denotes derivative with respect to the dimensionless space (time) variable. The spatial coordinate is normalized by the beam length $\ell$ and the time by $2 \pi / \bar{\omega}$ where $\bar{\omega}^{2}=E I_{b} / \rho \ell^{4}$. Here $E$ is Young's modulus, $\rho$ the mass/length for the material of the beam and $I_{b}$ the area moment of inertia about the bending axis.

By comparing (7) with (1) 1 and (4) $)_{1}$ we get

$$
L_{\mathrm{m}}(w):=w^{i v}, \quad D_{\mathrm{m}}(w):=\ddot{w}, \quad S_{\mathrm{m}}(k):=k^{4}, \quad T_{\mathrm{m}}(p):=p^{2} .
$$

Once suitable boundary conditions are chosen for the beam, the set $M_{\mathrm{m}}$ of the admissible discrete wavelengths $\left\{\alpha_{j}, j=1,2, \ldots\right\}$ is determined; therefore the $m$-eigenfrequencies are

$$
s_{j}= \pm \mathrm{i} \alpha_{j}^{2}, \quad j=1,2, \ldots
$$

\subsection{2. e-system}

The transmission line is governed, in the Laplace domain, by the following equation for the dimensionless voltage $v$ :

$$
-v^{\prime \prime}+p f(p) v=0, \quad f(p)=\ell^{2} \bar{\omega} C Z(p \bar{\omega}),
$$

where $f(p)$ represents the dimensionless impedance, $C$ is the capacitance per unit length of the transmission line and $Z$ is the impedance per unit length. We note that the telegraph equation, which governs the standard transmission line, is recovered by assuming the impedance $Z$ to be a simple inductance (i.e., $\left.f(p)=A_{0} p\right)$. As shown in Vidoli and dell'Isola (2000) the standard transmission line can be tuned (i.e., choosing the value of the parameter $A_{0}$ ) to control only one $m$-eigenfrequency. The derivation of $(9)$ is given in Appendix $\mathrm{A}$; for the tuning procedure the line is assumed to be non-dissipative, that is $R \rightarrow \infty$. However, some dissipative cases will be discussed for finite values of $R$; indeed optimal values of the resistance $R$ are sufficiently high not to affect the tuning effectiveness while dissipating the electrical energy.

A comparison of (9) with $(1)_{2}$ and $(4)_{2}$ gives

$$
L_{\mathrm{e}}(v):=-v^{\prime \prime}, \quad S_{\mathrm{e}}(k):=k^{2}, \quad F(p):=-p f(p) .
$$

Once suitable boundary conditions are chosen for the transmission line, the set $M_{\mathrm{e}}$ of the admissible discrete wavelengths $\left\{\beta_{j}, j=1,2, \ldots\right\}$ is determined; therefore the $e$-eigenfrequencies are

$$
\sigma_{j}=\left\{p: p f(p)=-\beta_{j}^{2}\right\}
$$

Note that depending upon the order of the rational function $f(p)$ each electric mode shape can have more than one resonant frequency. 
Problem 1 is now reformulated as follows.

Problem 2. Let $\widetilde{M}_{\mathrm{m}} \subset M_{\mathrm{m}}$ be the set of $n$ mechanical mode shapes to be controlled and $\left(\alpha_{h}, s_{h}\right), h \in \widetilde{M}_{\mathrm{m}}$ be their wavelengths and eigenfrequencies. Let $g: \widetilde{M}_{\mathrm{m}} \rightarrow M_{\mathrm{e}}$ be a one to one correspondence between the chosen mechanical modes and the electrical modes. Find the impedance $f(p)$ such that

$$
s_{h} \subset \sigma_{g(h)} \text { for every } h \in \widetilde{M}_{\mathrm{m}} \text {, }
$$

which, in view of (8) and (11) is equivalent to

$$
\mathrm{i} \alpha_{h}^{2} f\left(\mathrm{i} \alpha_{h}^{2}\right)=-\beta_{g(h)}^{2} .
$$

The correspondence $g$ can be obtained by maximizing the inner product between the electrical mode shape and the image under the coupling operator of the mechanical mode shape.

\section{Tuning procedure}

For $f(p)$ to be the impedance of a LC TTN (inductor-capacitor two-terminal network), it must be a real and positive function representable as

$$
f(p)=p \frac{A_{0} \prod_{i=1}^{n-1}\left(z_{i}^{2}+p^{2}\right)}{\prod_{i=1}^{n-1}\left(p_{i}^{2}+p^{2}\right)}, \quad A_{0}, z_{i}, p_{i} \in \mathbb{R},
$$

where

$$
z_{1}<p_{1}<z_{2}<p_{2}<z_{3}<p_{3} \cdots
$$

Therefore, Problem 2 is equivalent to Problem 3 defined below.

Problem 3. Find a rational function $f(p)$ satisfying conditions (13) and (14) and interpolating $n$ points $\left(\mathrm{i} \alpha_{h}^{2}, \frac{-\beta_{g(h)}^{2}}{\mathrm{i} \alpha_{h}^{2}}\right)$ where $g$ is the correspondence relation defined in Problem 2 and $h \in \widetilde{M}_{\mathrm{m}}$.

The following results on interpolation by rational functions (see Walsh, 1960) are essential to solve Problem 3.

Theorem 1. Let points $\zeta_{1}, \zeta_{2}, \ldots, \zeta_{m} \in \mathbb{C}$, not necessarily distinct, be given. Here $\mathbb{C}$ is the set of complex numbers. Also, let points $\eta_{1}, \eta_{2}, \ldots, \eta_{m+1} \in \mathbb{C}$ distinct from $\zeta_{k}$ but not necessarily distinct from each other, and values $\mu_{1}, \mu_{2}, \ldots, \mu_{m+1} \in \mathbb{C}$ be given. Then there exists a unique rational function, $r(p)$, of the form

$$
\frac{b_{0} p^{m-1}+b_{1} p^{m-2}+\cdots+b_{m}}{\left(p-\zeta_{1}\right)\left(p-\zeta_{2}\right) \cdots\left(p-\zeta_{m}\right)}
$$

which takes on the values $\mu_{k}$ at the points $\eta_{k}$.

Corollary 1. If $\eta_{1} \neq \eta_{2} \cdots \neq \eta_{m+1}$, then the rational function, $r(p)$, is given by

$$
r(p)=\sum_{k=1}^{m+1} \mu_{k} \frac{\omega(p)}{\left(p-\eta_{k}\right) \omega^{\prime}\left(\eta_{k}\right)},
$$

where

$$
\omega(p)=\frac{\left(p-\eta_{1}\right)\left(p-\eta_{2}\right) \cdots\left(p-\eta_{m+1}\right)}{\left(p-\zeta_{1}\right)\left(p-\zeta_{2}\right) \cdots\left(p-\zeta_{m}\right)} .
$$


Hence, to find a solution $f(p)$ of Problem 3, we use the interpolation formula (16) with

$$
\zeta_{k}= \pm \mathrm{i} p_{k}, \quad \eta_{k}= \pm \mathrm{i} \alpha_{k}^{2}, \quad \mu_{k}=\left(\frac{\beta_{g(k)}}{\alpha_{k}^{2}}\right)^{2}, \quad m=2 n-1,
$$

where $n$ is the number of mechanical modes to be controlled. With some arrangement we obtain

$$
\frac{f(p)}{p}=\sum_{h=1}^{n} \omega(p)\left(\frac{\beta_{g(h)}}{\alpha_{h}^{2}}\right)^{2}\left[\frac{1}{\left(p+\mathrm{i} a\left(\alpha_{h}\right)^{2}\right) \omega^{\prime}\left(-\mathrm{i} a\left(\alpha_{h}\right)^{2}\right)}+\frac{1}{\left(p-\mathrm{i} a\left(\alpha_{h}\right)^{2}\right) \omega^{\prime}\left(\mathrm{i} a\left(\alpha_{h}\right)^{2}\right)}\right]
$$

with

$$
\omega(p)=\frac{\left(p^{2}+\alpha_{1}^{4}\right)\left(p^{2}+\alpha_{2}^{4}\right) \cdots\left(p^{2}+\alpha_{n+1}^{4}\right)}{\left(p^{2}+p_{1}^{2}\right)\left(p^{2}+p_{2}^{2}\right) \cdots\left(p^{2}+p_{n}^{2}\right)} .
$$

The function $f(p)$ given by (19) trivially satisfies (13). In order to also satisfy condition (14) we choose poles $p_{k}$ interlaced with the values of $\eta_{k}$. In particular, we assume that

$$
p_{k}=\frac{\eta_{k+1}+\eta_{k}}{2}, \quad k=1,2, \ldots, n-1 .
$$

\subsection{Remarks on eigenfrequencies of the electric circuit}

In this section a further insight on eigensystem of the electric circuit is provided. The dispersive relation (11) for the $e$-system and the form (13) for the function $f(p)$ give

$$
p^{2} \frac{A_{0} \prod_{i=1}^{n-1}\left(z_{i}^{2}+p^{2}\right)}{\prod_{i=1}^{n-1}\left(p_{i}^{2}+p^{2}\right)}+\beta^{2}=0 .
$$

For each value of $\beta$, we obtain $2 n$ solutions for the complex frequency $p$. For high values of the electric wavelength $\beta$ we note that

$$
\left\{p: \prod_{i=1}^{n-1}\left(p_{i}^{2}+p^{2}\right)=0\right\} \subset \lim _{\beta \rightarrow+\infty}\left\{p: p^{2} \frac{A_{0} \prod_{i=1}^{n-1}\left(z_{i}^{2}+p^{2}\right)}{\prod_{i=1}^{n-1}\left(p_{i}^{2}+p^{2}\right)}+\beta^{2}=0\right\},
$$

i.e., $2(n-1)$ of the $2 n$ solutions of the dispersive relations converge to the limit values given by the poles of $f(p)$.

For $n=3$, Fig. 2 evinces qualitatively the eigenstructure. For

$$
f(p)=\frac{P(p)}{\left(p^{2}+\alpha_{1}^{2}\right)\left(p^{2}+\alpha_{2}^{2}\right)},
$$

we have plotted as solid vertical lines positions of the electric eigenfrequencies. We can easily recognize three (equal to the number $n$ of controlled modes) different clusters. The first two clusters have as accumulation points the two poles of the function $f(p)$, while the accumulation point of the last one is at $+\infty$.

As shown in Fig. 2, to each mode shape corresponds an eigenfrequency in each cluster; more precisely to the $j$ th mode shape corresponds the $j$ th eigenfrequency in all clusters.

This behavior of the electric system has important consequences in a numerical solution of the evolution problem (see for instance example 3), and will be taken into account in the subsequent modal analysis. More precisely, the determination of the response of the system to forcing terms whose frequency band is near the accumulation points requires a careful analysis since many different mode shapes can be excited within a very small frequency range. However, if the forcing term has a sufficiently smooth distribution 


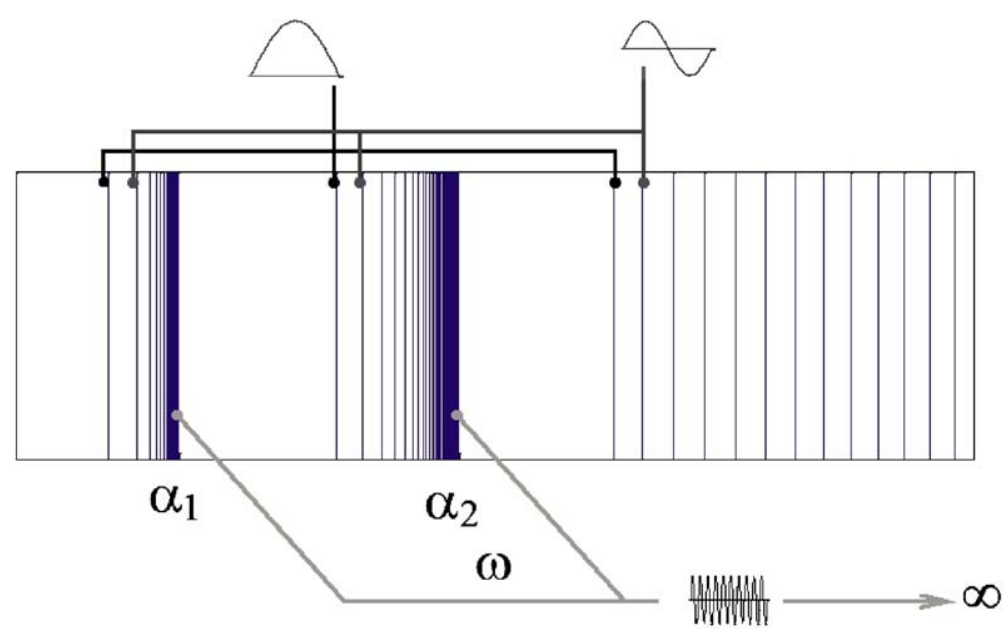

Fig. 2. For $n=3$, eigenfrequencies of the electric system are indicated by solid vertical lines; the accumulation point of the third cluster is at $+\infty$. The corresponding mode shapes are depicted at the top of the figure.

along the beam, its projection onto the mode shapes involved in the given frequency range is very small and can be neglected.

The procedure to find the impedance of a LC TTN is given in Appendix C.

\section{Applications}

The effectiveness of the designed device as a damper of mechanical vibrations is now illustrated by three examples differing in the prescribed boundary conditions. In each example more than one mode of vibration is controlled simultaneously in order to demonstrate the effectiveness of the proposed method. We note that the aforementioned tuning procedure is based on the analysis of the uncoupled system. Once the interpolation problem has been solved and the multiresonant circuit has been synthesized, the electrical and the mechanical systems are coupled through the piezoelectric actuators and the evolution of the entire system is investigated.

In terms of non-dimensional variables, deformations of these electromechanical systems are governed, in the Laplace domain, by

$$
\left\{\begin{array}{l}
p^{2} w+w^{i v}-\gamma p \psi^{\prime \prime}-p w_{0}-v_{0}=0 \\
p f(p) \psi-\psi^{\prime \prime}+\gamma f(p) w^{\prime \prime}-\frac{f(p)}{p} w_{0}^{\prime \prime}=0
\end{array}\right.
$$

where $\psi$ is the dimensionless electric flux linkage (i.e., a time derivative of the electric potential), $\gamma=k_{m e} / \ell \sqrt{\rho}$ is the dimensionless piezoelectric coupling coefficient and

$$
w_{0}(\xi)=w(\xi, 0), \quad v_{0}(\xi)=\dot{w}(\xi, 0) .
$$

For all problems studied a Galerkin procedure will be used by considering the following expansions:

$$
w(\xi, p)=\mathbf{N}(\xi) \mathbf{W}(p), \quad \psi(\xi, p)=\mathbf{n}(\xi) \mathbf{\Psi}(p), \quad w_{0}(\xi)=\mathbf{N}(\xi) \mathbf{W}^{0}, \quad v_{0}(\xi)=\mathbf{N}(\xi) \mathbf{V}^{0}
$$

where $\mathbf{N}(\xi)$ and $\mathbf{n}(\xi)$ are vectors of suitable basis functions, while $\mathbf{W}(p), \boldsymbol{\Psi}(p), \mathbf{W}^{0}$ and $\mathbf{V}^{0}$ are the associated coefficients for the mechanical and the electrical mode shapes. Eq. (24) reduce to the following algebraic system for the unknowns $\mathbf{W}(p)$ and $\boldsymbol{\Psi}(p)$ : 


$$
\left\{\begin{array}{l}
p^{2} \mathbf{M}_{\mathrm{m}} \mathbf{W}(p)+\mathbf{K}_{\mathrm{m}} \mathbf{W}(p)-\gamma p \mathbf{C} \boldsymbol{\Psi}(p)-p \mathbf{M}_{\mathrm{m}} \mathbf{W}^{0}+\mathbf{M}_{\mathrm{m}} \mathbf{V}^{0}=\mathbf{0} \\
p f(p) \mathbf{M}_{\mathrm{e}} \boldsymbol{\Psi}(p)-\mathbf{K}_{\mathrm{e}} \boldsymbol{\Psi}(p)+\gamma f(p) \mathbf{C}^{\mathrm{T}} \mathbf{W}(p)-\frac{f(p)}{p} \mathbf{C}^{\mathrm{T}} \mathbf{W}^{0}=\mathbf{0}
\end{array}\right.
$$

where

$$
\begin{aligned}
& \mathbf{M}_{\mathrm{m}}=\int_{0}^{1} \mathbf{N}(\xi)^{\mathrm{T}} \mathbf{N}(\xi) \mathrm{d} \xi, \quad \mathbf{K}_{\mathrm{m}}=\int_{0}^{1} \mathbf{N}^{\prime \prime}(\xi)^{\mathrm{T}} \mathbf{N}^{\prime \prime}(\xi) \mathrm{d} \xi, \quad \mathbf{C}=\int_{0}^{1} \mathbf{N}^{\prime}(\xi)^{\mathrm{T}} \mathbf{n}^{\prime}(\xi) \mathrm{d} \xi \\
& \mathbf{M}_{\mathrm{e}}=\int_{0}^{1} \mathbf{n}(\xi)^{\mathrm{T}} \mathbf{n}(\xi) \mathrm{d} \xi, \quad \mathbf{K}_{\mathrm{e}}=\int_{0}^{1} \mathbf{n}^{\prime}(\xi)^{\mathrm{T}} \mathbf{n}^{\prime}(\xi) \mathrm{d} \xi
\end{aligned}
$$

The coupling between the coefficients of the mechanical and the electrical mode shapes is determined by the matrix $\mathbf{C}$; thus this matrix determines the choice of the one-to-one correspondence $g$ introduced in Problem 2. $\mathbf{M}_{\mathrm{m}}$ and $\mathbf{K}_{\mathrm{m}}$ are, respectively, the mass matrix and the stiffness matrix for the mechanical problem.

We note that in some cases Eq. (27) can be solved analytically, but in general we must seek numerical solutions even when the uncoupled governing equations have closed form solutions.

\subsection{Simply supported beam}

The mechanical boundary conditions for the simply supported beam are

$$
w(0, p)=0, \quad w(1, p)=0, \quad w^{\prime \prime}(0, p)=0, \quad w^{\prime \prime}(1, p)=0 .
$$

In order to get the same mode shapes for the electrical system, the two ends of the electric line must be grounded; that is

$$
\psi(0, p)=0, \quad \psi(1, p)=0 .
$$

Let us assume that the first four modes of vibration are to be controlled. Thus the rational function $f(p)$ will have six poles and seven zeroes. For the chosen boundary conditions, the $h$ th mechanical and electrical mode shapes are equal, and the correspondence function $g$ is trivially defined as $g(h)=h$.

Fig. 3 shows the function $f(p) / p$ and the dimensionless impedance $f(p)$ for $p=\mathrm{i} \omega$. The frequency response functions (FRFs) of the tuned uncoupled mechanical and electrical systems are exhibited in Fig. 4. It is clear that the first four frequencies of the mechanical system coincide with four of the resonance frequencies

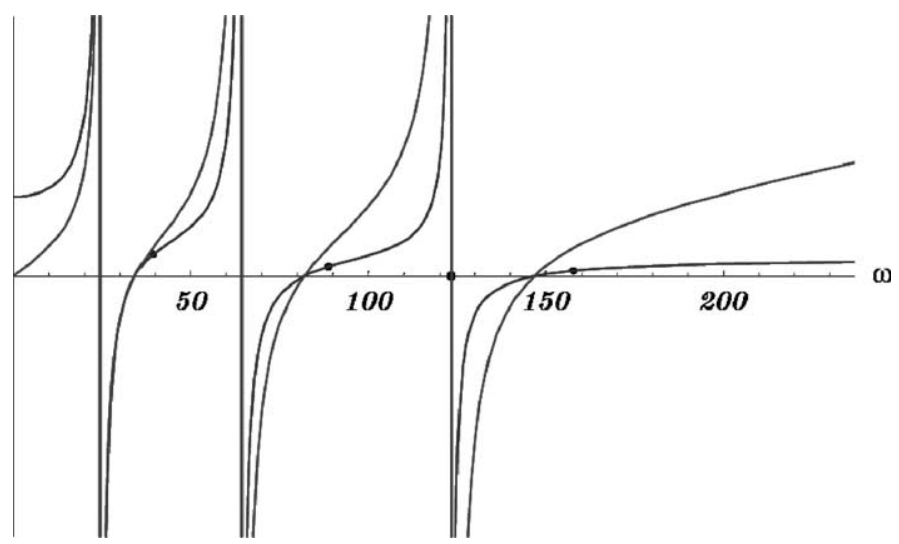

Fig. 3. Solid curves illustrate the function $f(p) / p$ interpolating the points corresponding to the ordered set (mechanical wavelength, mechanical eigenfrequency) and having given poles; dashed curves show the computed impedance $f(p)$. Both are evaluated on the imaginary axis, i.e. for $p=\mathrm{i} \omega$. Dots are poles of the function $f(p)$. 


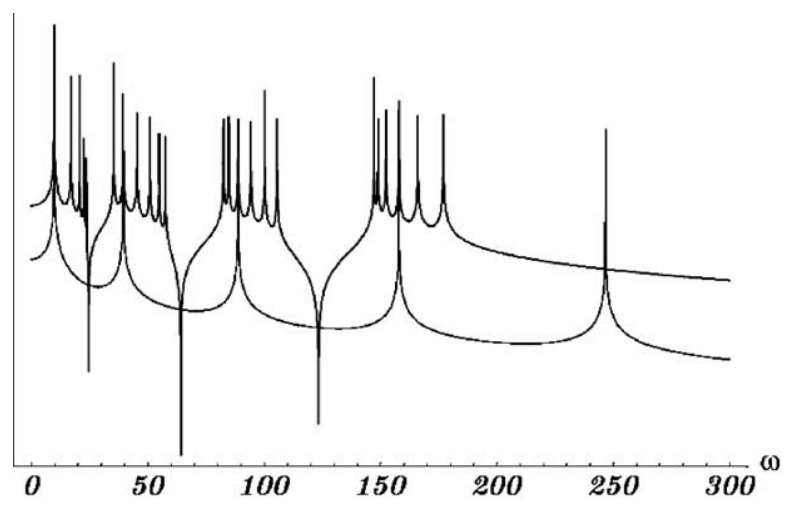

Fig. 4. Frequency response functions of the electrical (solid) and the mechanical (dashed) systems.

of the electrical system. The large bandwidth of the electrical FRF for the designed system, as depicted in Fig. 4, reveals the robustness of the proposed technique. The electric FRF (solid curve) has a relevant bandwidth around every peak of the mechanical FRF (dashed curve). Hence, if the frequency of a given mechanical mode, say $h$, is consistently mismatched, this mode can possibly be coupled with an electrical mode $h \pm 1$. In this case, the efficiency of the system in damping out vibrations is not noticeably affected. In order to overcome systematic mismatching of all mechanical modes, it is better to adaptively tune the electric subsystem.

The eigenfunctions of the uncoupled systems are chosen as the basis functions:

$$
N_{j}(\xi)=n_{j}(\xi)=\sin \left(\alpha_{j} \xi\right), \quad \alpha_{j}:=j \pi, \quad j=1,2, \ldots
$$

The system (27) becomes

$$
\left\{\begin{array}{l}
p^{2} W_{j}(p)+\alpha_{j}^{4} W_{j}(p)+\alpha_{j}^{2} \gamma p \Psi_{j}(p)-p \delta_{j h}=0, \\
p f(p) \Psi_{j}(p)+\alpha_{j}^{2} \Psi_{j}(p)-\alpha_{j}^{2} \gamma p W_{j}(p)+\alpha_{h}^{2} \frac{f(p)}{p} \delta_{j h}=0, \quad j=1, \ldots, n,
\end{array}\right.
$$

where $\delta_{j h}$ is the Kronecker delta and we have taken $v_{0}=0$ and $w_{0}=\sin \alpha_{h} \xi$. Solving the system (32) for the unknown functions $W_{j}(p)$ and $\Psi_{j}(p)$ we get the following non-trivial solutions only for $j=h$.

$$
\begin{aligned}
& W_{j}(p)=\frac{\alpha_{j}^{2} p+\left(p^{2}+\alpha_{j}^{4} \gamma^{2}\right) f(p)}{\alpha_{j}^{2}\left(\alpha_{j}^{4}+p^{2}\right)+p\left(p^{2}+\alpha_{j}^{4}\left(1+\gamma^{2}\right)\right) f(p)}, \\
& \Psi_{j}(p)=-\frac{\alpha_{j}^{6} \gamma f(p)}{p\left(\alpha_{j}^{2}\left(\alpha_{j}^{4}+p^{2}\right)+p\left(p^{2}+\alpha_{j}^{4}\left(1+\gamma^{2}\right)\right)\right) f(p)} .
\end{aligned}
$$

The inverse Laplace transform of these expressions provides the time dependence of the modal coefficients.

Fig. 5a exhibits, for the undamped case (i.e., $R \rightarrow \infty$ ) the time evolution of the modal coefficients for initial conditions on each of the first four mechanical modes, as well as the time history of the energy content of both systems. There is no spill-over effect; that is, given an initial condition on the $j$ th mechanical mode, the electromechanical coupling will occur only between the $j$ th mechanical and the $j$ th electrical modes. In Fig. 5b, we have plotted results similar to those of Fig. 5a but with a classical transmission line (i.e., $\left.f(p)=A_{0} p\right)$ tuned on the first mechanical mode. Whereas the classical transmission line and the presently proposed circuit perform equally well in terms of the energy exchanged between the mechanical and the electrical systems for the first vibration mode, for the other modes the present device performs considerably better. This is because the present device has been tuned to the first four mechanical modes. Fig. 6a and $b$ show the time evolutions of the mechanical and the electrical systems with damping added to the 

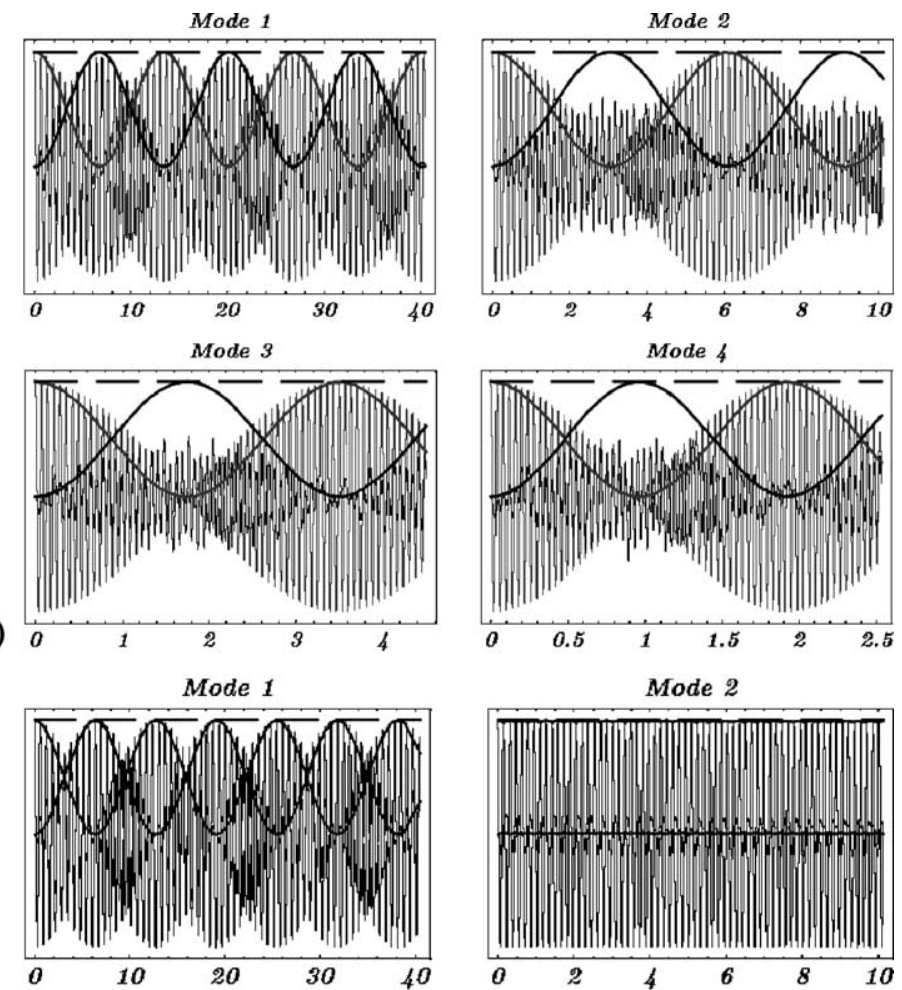

Mode 2

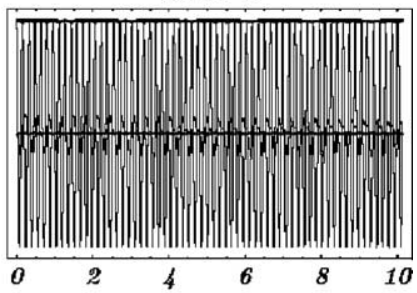

Mode 3

Mode 4
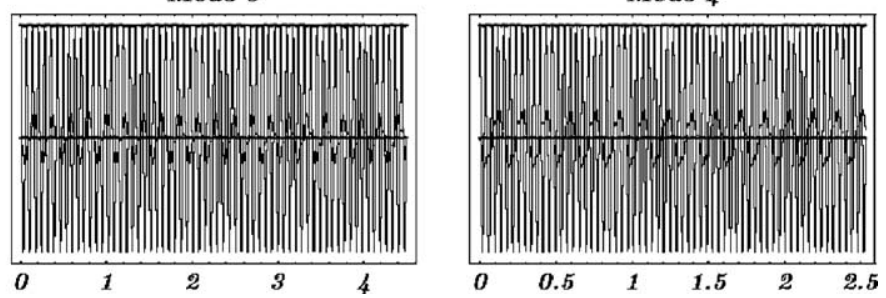

Fig. 5. For the undamped case, the time evolution of the electrical (dark) and the mechanical (grey) systems, starting from initial conditions on the first four mechanical modes. Thin and thick lines represent modal coefficients and energy content respectively. The dashed line represents the total energy of the system. The dimensionless time has been chosen so that the time period of the first mode of the simply supported beam equals 1. (a) LC TTN circuit optimized according to the present work; (b) standard transmission line tuned on the first mechanical mode.

proposed device and the classical transmission line. Again, the proposed device performs much better than the classical transmission line.

\subsection{Clamped-clamped beam}

In the case of a clamped-clamped beam we cannot provide a semi-analytical solution as was done in the previous example. Now boundary conditions are

$$
w(0, p)=0, \quad w^{\prime}(0, p)=0, \quad w(1, p)=0, \quad w^{\prime}(1, p)=0, \quad \psi(0, p)=0, \quad \psi(1, p)=0 .
$$



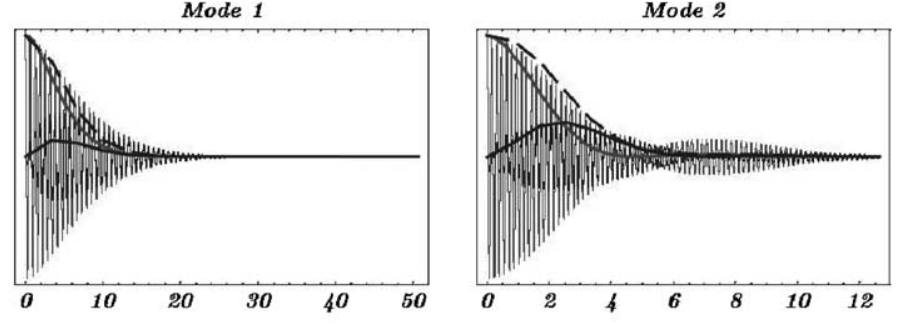

Mode 3

(a)

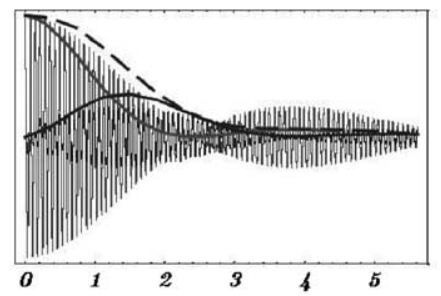

Mode 4
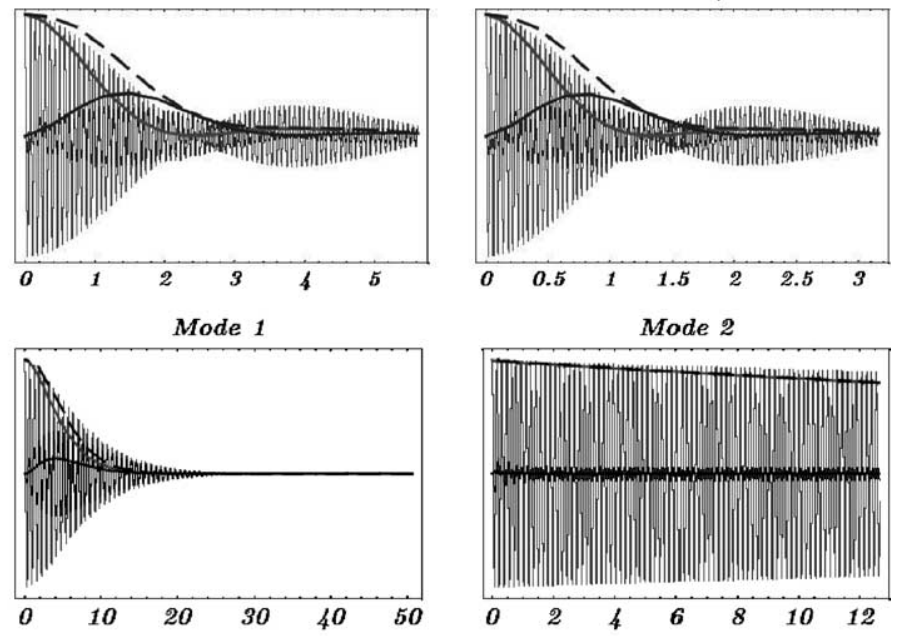

Mode 3
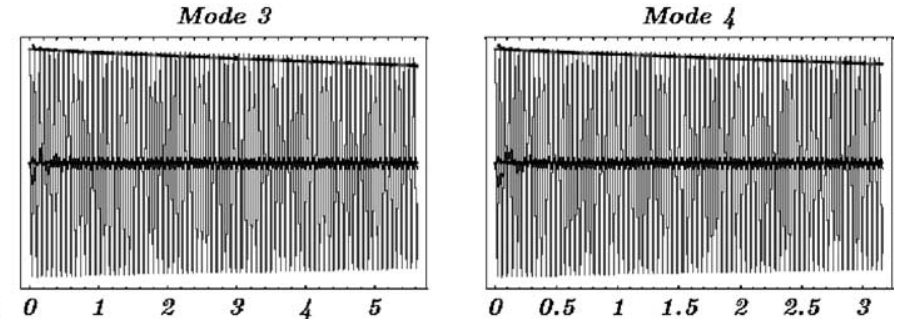

Fig. 6. For the damped case, the time evolution of the electrical (dark) and the mechanical (grey) systems, starting from initial conditions on the first four mechanical modes. Thin and thick lines represent modal coefficients and energy content respectively. The dashed line represents the total energy of the system. (a) LC TTN circuit optimized according to the present work; (b) standard transmission line tuned on the first mechanical mode.

We assume that the first two modes of vibration are to be controlled so that the rational function $f(p)$ will have two poles and three zeroes. The basis functions are chosen to satisfy the uncoupled eigenvalue problems:

$$
\begin{aligned}
& N_{i}(\xi)=c_{1} \sin \left(\alpha_{i} \xi\right)+c_{2} \cos \left(\alpha_{i} \xi\right)+c_{3} \sinh \left(\alpha_{i} \xi\right)+c_{4} \cosh \left(\alpha_{i} \xi\right), \\
& n_{i}(\xi)=\sin \left(\beta_{i} \xi\right) .
\end{aligned}
$$

The system (27) becomes

$$
\left\{\begin{array}{l}
p^{2} W_{j}(p)+\alpha_{j}^{4} W_{j}(p)+\gamma p C_{i j} \Psi_{i}(p)-p W_{j}^{0}+V_{j}^{0}=0, \\
p f(p) \Psi_{j}(p)+\beta_{j}^{2} \Psi_{j}(p)+\gamma p C_{j i} W_{i}(p)-\gamma \frac{f(p)}{p} C_{j i} W_{i}^{0}=0, \quad j=1, \ldots, n .
\end{array}\right.
$$


Mode 1

(a)

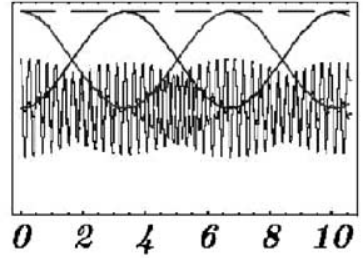

Mode 1

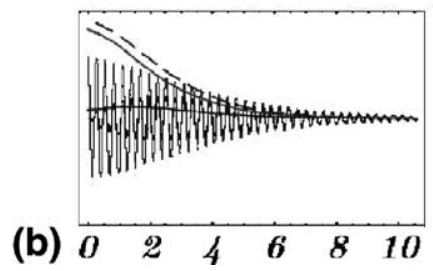

Mode 2

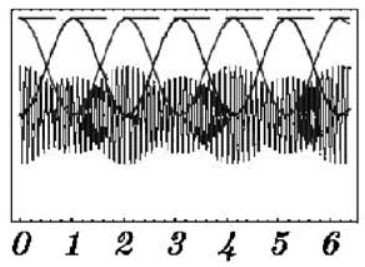

Mode 2

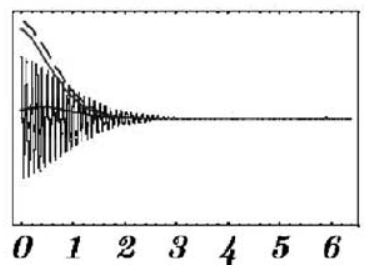

Mode 3

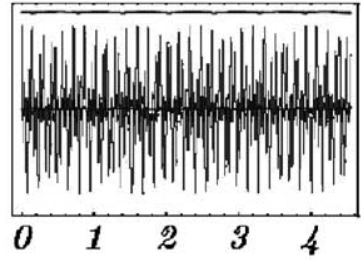

Mode 3

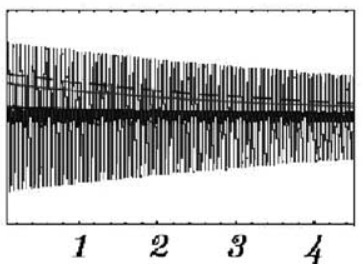

Fig. 7. Time evolution of the electrical (dark) and mechanical (grey) systems, starting from initial conditions on the first two mechanical modes. Thin and thick lines represent modal coefficients and energy content respectively. The dashed line represents the total energy of the system; (a) undamped, (b) damped.

The coupling matrix $C$ is quasi-diagonal; this means that the projection of the $h$ th mechanical mode on all electric modes has the most relevant contribution only along the $h$ th electric mode; thus the correspondence function $g$ is again defined as $g(h)=h$.

Eq. (35) is solved numerically. Fig. 7a and b exhibit, for the undamped and the damped cases, respectively, the time evolution of the modal coefficients for initial conditions on each of the first two mechanical modes. As in the previous example, the proposed device performs very well in controlling the two modes of vibration of the beam. The time evolution of the energy content shown in Fig. 7a provides a proof (necessary but not sufficient) of the efficiency of the used modal expansion.

\subsection{Cantilever beam}

The proposed technique is now applied to a cantilever beam. As already mentioned, the tuning procedure is based on the uncoupled systems; however, in this case the modal expansion used in the previous two examples does not provide a convenient solution. In fact the boundary conditions to be used in the present application are such that the use of purely electrical and mechanical vibration modes in Fourier expansion does not allow an efficient approximation of the solutions of the coupled system. Therefore, a standard finite element technique is used to analyze the problem.

The governing equations (27) for the coupled system are complemented by the following boundary conditions for a cantilever beam (the electric boundary conditions are chosen in order to maximize the electromechanical coupling):

$$
w(0, p)=w^{\prime}(0, p)=w^{\prime \prime}(1, p)=w^{\prime \prime \prime}(1, p)=\psi^{\prime}(0, p)=\psi(1, p)=0 .
$$

We now apply the tuning procedure to find the function $f(p)$ and synthesize the multiresonant electric circuit. For this example we assume that the first three modes of vibration are to be controlled.

Since our goal is to tune the electric system to the first three mechanical frequencies, the rational function $f(p)$ will have four poles and five zeroes.

The procedure to numerically solve Eq. (27) with boundary conditions (36) requires a special technique and is described in Appendix B. 
Mode 1

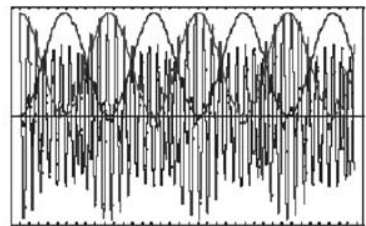

(a) 010203040506070

Mode 1

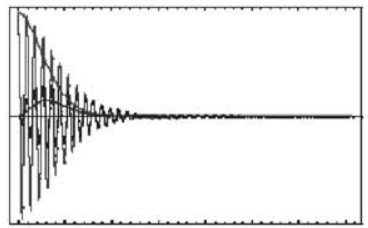

(b) 010203040506070
Mode 2

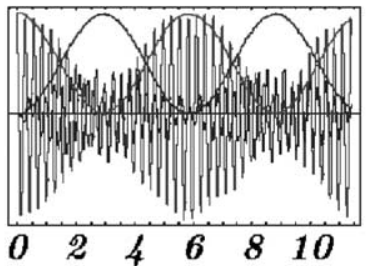

Mode 2

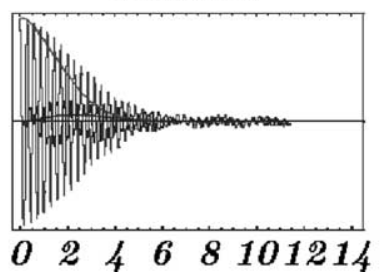

Mode 3

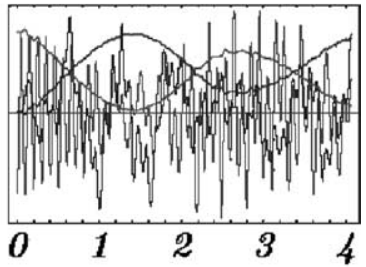

Mode 3

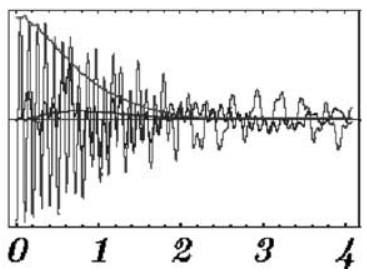

Fig. 8. Time evolution of the electrical (dark) and the mechanical (grey) systems, starting from initial conditions on the first three mechanical modes. Thin and thick lines represent the kinematical descriptors (flux linkage and displacement) of a particular point of the beam and the energy content respectively; (a) undamped, (b) damped.

For damping factor equal to zero, Fig. 8a exhibits the time evolution of the mechanical and the electrical systems and of the electrical and the mechanical energy content, starting from initial conditions given on the first three mechanical vibration modes. The strange evolution of the third mode is due to the "spill-over effect" towards electrical eigenmodes. In fact the first and the second electric vibration modes have one of their eigenfrequencies very close to the eigenfrequency of the third mechanical vibration mode, which is therefore coupled with the first three electric modes. Therefore, the energy initially provided to the third mechanical mode is distributed over the first three mechanical modes by means of the electric system. We have plotted results in Fig. 8b for a system with numerically optimized damping factor. As expected, the proposed device is actually capable of controlling and damping mechanical vibrations in the desired modal range.

\section{Conclusions}

We have designed an electromechanical system comprised of a beam, piezoceramic actuators and a passive electrical network containing inductors, capacitors and resistors. The electrical system, comprised of the piezoceramic patches affixed to the top and/or the bottom surfaces of the beam and the electric network, has been modeled as a continuous system. It is reasonable to do so because of the large wavelengths of the first few flexural modes of the beam relative to the distance between the patches. The impedance of the network is selected to simultaneously control more than one mode of flexural vibration of the beam. Three examples are given to demonstrate the effectiveness of the proposed control technique. In the first one we simultaneously control first four flexural modes of a simply supported beam, and a semi-analytical solution has been found. In the second one, we control the first two flexural modes of a clamped-clamped beam, and a numerical solution, via modal projection, has been found. In the last example, we simultaneously control first three flexural modes of a cantilever beam, and numerical solutions, via a newly designed FE-Runge-Kutta code, have been found. In all three examples the proposed device performs excellently as a multimode vibration damper. 


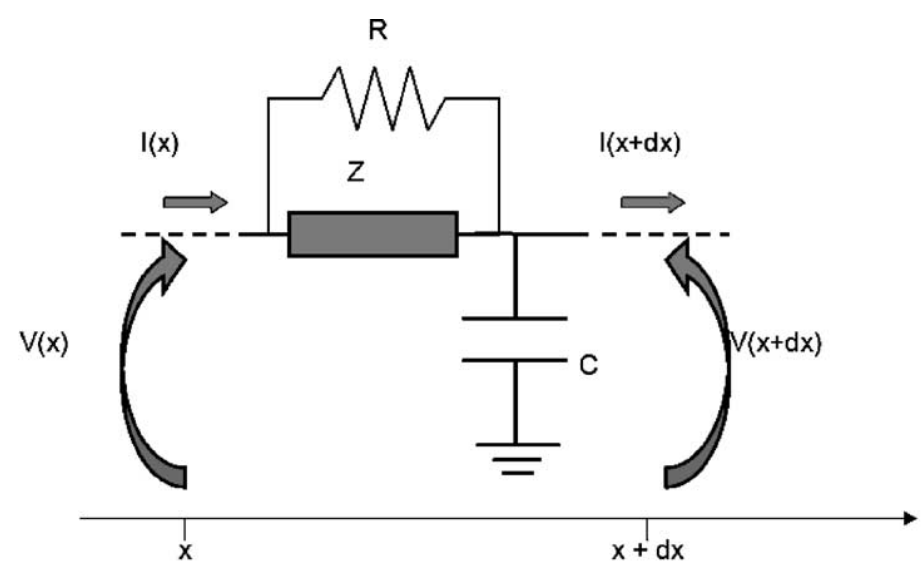

Fig. 9. Single element of the modular electric line.

\section{Appendix A. Derivation of governing equations of the $e$-system}

For deriving the equation giving the flow of current in the electric circuit, an element of the circuit is depicted in Fig. 9. The governing equations for this circuit are

$$
\begin{aligned}
& I(x+\mathrm{d} x)-I(x)=-p C V(x) \mathrm{d} x, \\
& V(x+\mathrm{d} x)-V(x)=-H(p) I(x) \mathrm{d} x .
\end{aligned}
$$

Here $I$ is the current, $V$ the voltage, $C$ the capacitance of the PZT, $p$ is the dimensionless Laplace transform variable, $Z(p)$ the electric impedance, and

$$
H(p)=\frac{R Z(p)}{R+Z(p)} .
$$

In the non-dissipative case (i.e. when the resistance $R \rightarrow \infty) H(p)=Z(p)$.

In the limiting case of $\mathrm{d} x \rightarrow 0$, Eqs. (37) and (38) become

$$
I^{\prime}=-p C V, \quad V^{\prime}=-H(p) I,
$$

which upon elimination of $I$ and the introduction of the following non-dimensional variables

$$
v=\frac{V}{V^{*}}, \quad p=\frac{s}{\bar{\omega}}, \quad f(p)=L^{2} \bar{\omega} C H(p \bar{\omega})
$$

give

$$
v^{\prime \prime}-p f(p) v=0 .
$$

Here $L$ is a characteristic inductance/length and $V^{*}$ a characteristic voltage.

\section{Appendix B. Details of numerical solution of Eqs. (27) and (36)}

In order to numerically solve the problem defined by Eq. (27) and boundary conditions (36), we introduce its representation in the time domain. For this purpose, we consider a general form of the function $f(p)$ 


$$
f(p)=\frac{n_{1} p+n_{3} p^{3}+\cdots+n_{2 r-1} p^{2 r-1}}{d_{0}+d_{2} p^{2}+\cdots+d_{2 r-2} p^{2 r-2}+\delta\left(n_{1} p+n_{3} p^{3}+\cdots+n_{2 r-1} p^{2 r-1}\right)}
$$

where $r$ is the number of mechanical modes to be controlled and $\delta$ is the damping factor. Multiplying (27) by the denominator of $f(p)$ and arranging terms we obtain

$$
\begin{aligned}
& \mathbf{M}_{\mathrm{e}} n_{2 r-1} p^{2 r} \boldsymbol{\Psi}(p)+\left(\mathbf{M}_{\mathrm{e}} \sum_{i=1}^{r-1} n_{2 i-1} p^{2 i}-\mathbf{K}_{\mathrm{e}} \sum_{i=1}^{r} d_{2 i-2} p^{2 i-2}\right) \boldsymbol{\Psi}(p)-\delta \mathbf{K}_{\mathrm{e}} \sum_{i=1}^{r} n_{2 i-1} p^{2 i-1} \boldsymbol{\Psi}(p) \\
& \quad+\gamma \mathbf{C}^{\mathrm{T}}\left(\sum_{i=1}^{r} n_{2 i-1} p^{2 i-1}\right) \mathbf{W}(p)=0,
\end{aligned}
$$

where initial conditions have not been considered since they will be taken into account in the subsequent time analysis. The inverse Laplace transform of (44) is

$$
\begin{aligned}
\mathbf{M}_{\mathrm{e}} n_{2 r-1} \frac{\mathrm{d}^{(2 r)}}{\mathrm{d} t^{2 r}} \boldsymbol{\Psi}(t)+\left(\mathbf{M}_{\mathrm{e}} \sum_{i=1}^{r-1} n_{2 i-1} \frac{\mathrm{d}^{(2 i)}}{\mathrm{d} t^{2 i}}-\mathbf{K}_{\mathrm{e}} \sum_{i=1}^{r} d_{2 i-2} \frac{\mathrm{d}^{(2 i-2)}}{\mathrm{d} t^{2 i}}\right) \boldsymbol{\Psi}(t)-\delta \mathbf{K}_{\mathrm{e}} \sum_{i=1}^{r} n_{2 i-1} \frac{\mathrm{d}^{(2 i-1)}}{\mathrm{d} t^{2 i-1}} \boldsymbol{\Psi}(t) \\
+\gamma \mathbf{C}^{\mathrm{T}}\left(\sum_{i=1}^{r} n_{2 i-1} \frac{\mathrm{d}^{(2 i-1)}}{\mathrm{d} t^{2 i}}\right) \mathbf{W}(t)=0 .
\end{aligned}
$$

Eq. $(27)_{1}$ gives

$$
\mathbf{M}_{\mathrm{m}} \frac{\mathrm{d}^{2}}{\mathrm{~d} t^{2}} \mathbf{W}(t)+\mathbf{K}_{\mathrm{m}} \mathbf{W}(t)-\gamma \mathbf{C} \frac{\mathrm{d}}{\mathrm{d} t} \boldsymbol{\Psi}(t)=0 .
$$

We define

$$
\widetilde{\boldsymbol{\Psi}}^{(2 j-1)} \equiv \frac{\mathrm{d}}{\mathrm{d} t} \boldsymbol{\Psi}^{(2 j-2)}, \quad \widetilde{\mathbf{W}}^{(2 j-1)} \equiv \frac{\mathrm{d}}{\mathrm{d} t} \mathbf{W}^{(2 j-2)}, \quad j=1,2, \ldots, r .
$$

From Eqs. (45) and (46), using definitions (47), we get

$$
\left\{\begin{aligned}
\frac{\mathrm{d}}{\mathrm{d} t} \widetilde{\boldsymbol{\Psi}}^{(2 r-1)}= & -\frac{1}{n_{2 r-1}} \sum_{i=1}^{r-1} n_{2 i-1} \widetilde{\boldsymbol{\Psi}}^{(2 i)}-\frac{1}{n_{2 r-1}} \mathbf{M}_{\mathrm{e}}^{-1} \mathbf{K}_{\mathrm{e}} \sum_{i=1}^{r} d_{2 i-2} \widetilde{\boldsymbol{\Psi}}^{(2 i-2)} \\
& +\delta \frac{1}{n_{2 r-1}} \mathbf{M}_{\mathrm{e}}^{-1} \mathbf{K}_{\mathrm{e}} \sum_{i=1}^{r} n_{2 i-1} \widetilde{\boldsymbol{\Psi}}^{(2 i-1)}-\gamma \frac{1}{n_{2 r-1}} \mathbf{M}_{\mathrm{e}}^{-1} \mathbf{C}^{\mathrm{T}} \sum_{i=1}^{r} n_{2 i-1} \widetilde{\mathbf{W}}^{(2 i-1)}, \\
\frac{\mathrm{d}}{\mathrm{d} t} \widetilde{\mathbf{W}}^{(2 r-1)}= & -\mathbf{M}_{\mathrm{m}}^{-1} \mathbf{K}_{\mathrm{m}} \mathbf{W}^{(2 r-2)}+\gamma \mathbf{M}_{\mathrm{m}}^{-1} \mathbf{C} \widetilde{\boldsymbol{\Psi}}^{(2 r-1)} .
\end{aligned}\right.
$$

Therefore we can state the problem in the following standard form:

$$
\dot{\mathbf{x}}=\mathbf{A x}, \quad \mathbf{x}(\mathbf{0})=\mathbf{x}_{0},
$$

where

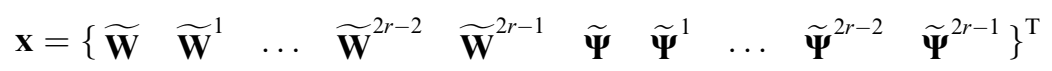

and $\mathbf{A}$ is the matrix of coefficients of Eqs. (47) and (48).

The system (49) of ordinary differential equations is numerically integrated with a Runge-Kutta algorithm. However, since its dimensions can be huge (if $n e$ is the number of elements in the mesh and $r$ is the number of mechanical modes to be controlled, $\mathbf{A}$ is a $\{6 r n e \times 6 r n e\}$ matrix), some preliminary reduction is mandatory. Since the matrix $\mathbf{A}$ is not symmetric, we must consider its right and left eigenvectors (arranged in the matrices $\mathbf{T}_{r}, \mathbf{T}_{l}$ ), which are defined by 


$$
\mathbf{A} \mathbf{T}_{r}^{\mathrm{T}}=\mathbf{T}_{r}^{\mathrm{T}} \lambda, \quad \mathbf{T}_{l} \mathbf{A}=\lambda \mathbf{T}_{l},
$$

where $\boldsymbol{\lambda}$ is the diagonal matrix of the eigenvalues of $\mathbf{A}$.

We define

$$
\mathbf{z}=\mathbf{T}_{l} \mathbf{x}, \quad \mathbf{x}=\mathbf{T}_{r} \mathbf{y},
$$

which implies that

$$
\mathbf{x}=\mathbf{T}_{r}\left(\mathbf{T}_{l} \mathbf{T}_{r}\right)^{-1} \mathbf{z} .
$$

Eq. (49) is then transformed into the following:

$$
\dot{\mathbf{z}}=\boldsymbol{\lambda} \mathbf{z}, \quad \mathbf{z}(\mathbf{0})=\mathbf{T}_{l} \mathbf{x}_{0} .
$$

Having found $\mathbf{z}, \mathbf{x}$ can be determined from Eq. (53).

Using the eigenvector representation (51) and a proper choice of a reduced subspace of eigenvectors, the system (54) of equations can be numerically solved. The choice of the eigenvectors to retain in the evolution equations is non-trivial. In fact, due to the high order electric dispersive relation, at low frequencies (namely, near the two accumulation points of Fig. 2) we still have countably many eigenvectors, with different mode shapes. Thus the range of selection of eigenvectors based on their frequencies is not enough to reduce the number of modes involved in the evolution of the system. To make an accurate selection a wavenumber filter is necessary. We retain only modes with a relevant projection onto a given finite dimensional functional space (for instance the space of polynomial on $\mathbb{R}$ with degree less than 2 ).

\section{Appendix C. Synthesis of the electrical impedance}

The rational function $Z(s)$ derived by using the tuning procedure of Section 3 represents the impedance of a LC TTN. We note that many different LC TTNs have the same equivalent impedance. Here we look for a particular LC TTN represented by the given rational function. We use the Cauer method summarized below. The impedance $Z(s)$ has the following form (see Eqs. (13) and (14)):

$$
Z(s)=s \frac{B_{0} \prod_{i=1}^{n-1}\left(\tilde{z}_{i}^{2}+s^{2}\right)}{\prod_{i=1}^{n-1}\left(\tilde{p}_{i}^{2}+s^{2}\right)}, \quad B_{0}, \tilde{z}_{i}, \tilde{p}_{i} \in \mathbb{R} .
$$

Hence its inverse

$$
Y(s)=Z^{-1}(s)=\frac{\prod_{i=1}^{n-1}\left(\tilde{p}_{i}^{2}+s^{2}\right)}{s B_{0} \prod_{i=1}^{n-1}\left(\tilde{z}_{i}^{2}+s^{2}\right)}=\frac{R_{1}}{s}+Y_{1}(s),
$$

is the admittance of a LC TTN. Here $R_{1}$ is the residue at $s=0$ of $Y(s)$ and $Y_{1}(s)$. Eq. (56) shows that $Y(s)$ is the admittance of an inductor (say $L_{1}$ ) of admittance $1 / R_{1}$ and connected in parallel with another LC TTN of admittance

$$
Y_{1}(s)=\frac{s B_{1} \prod_{i=1}^{n-2}\left(\tilde{p}_{1 i}^{2}+s^{2}\right)}{\prod_{i=1}^{n-1}\left(\tilde{z}_{1 i}^{2}+s^{2}\right)} .
$$

This can be inverted to get the corresponding impedance:

$$
Z_{1}(s)=\frac{\prod_{i=1}^{n-1}\left(\tilde{z}_{1 i}^{2}+s^{2}\right)}{s B_{1} \prod_{i=1}^{n-2}\left(\tilde{p}_{1 i}^{2}+s^{2}\right)}=\frac{\bar{R}_{1}}{s}+Z_{2}(s)
$$




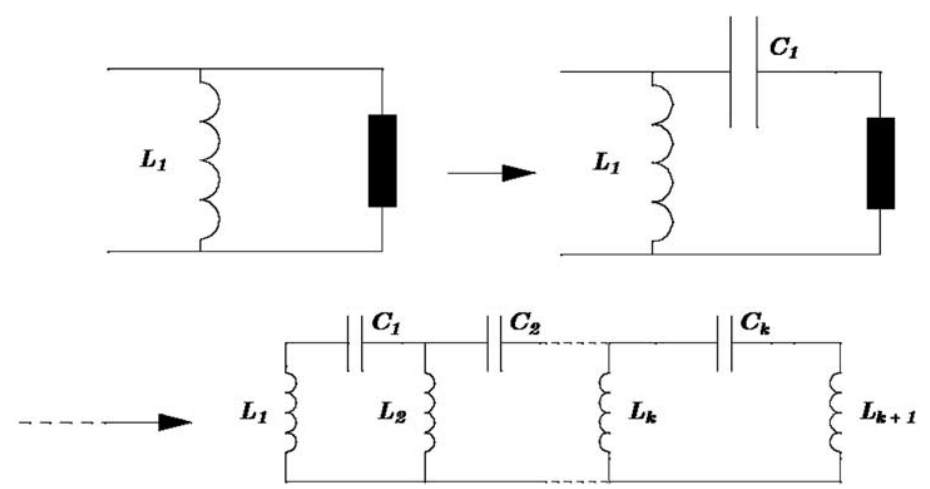

Fig. 10. Cauer iterative method for the synthesis of a LC TTN; arrows show the logical path between the steps.
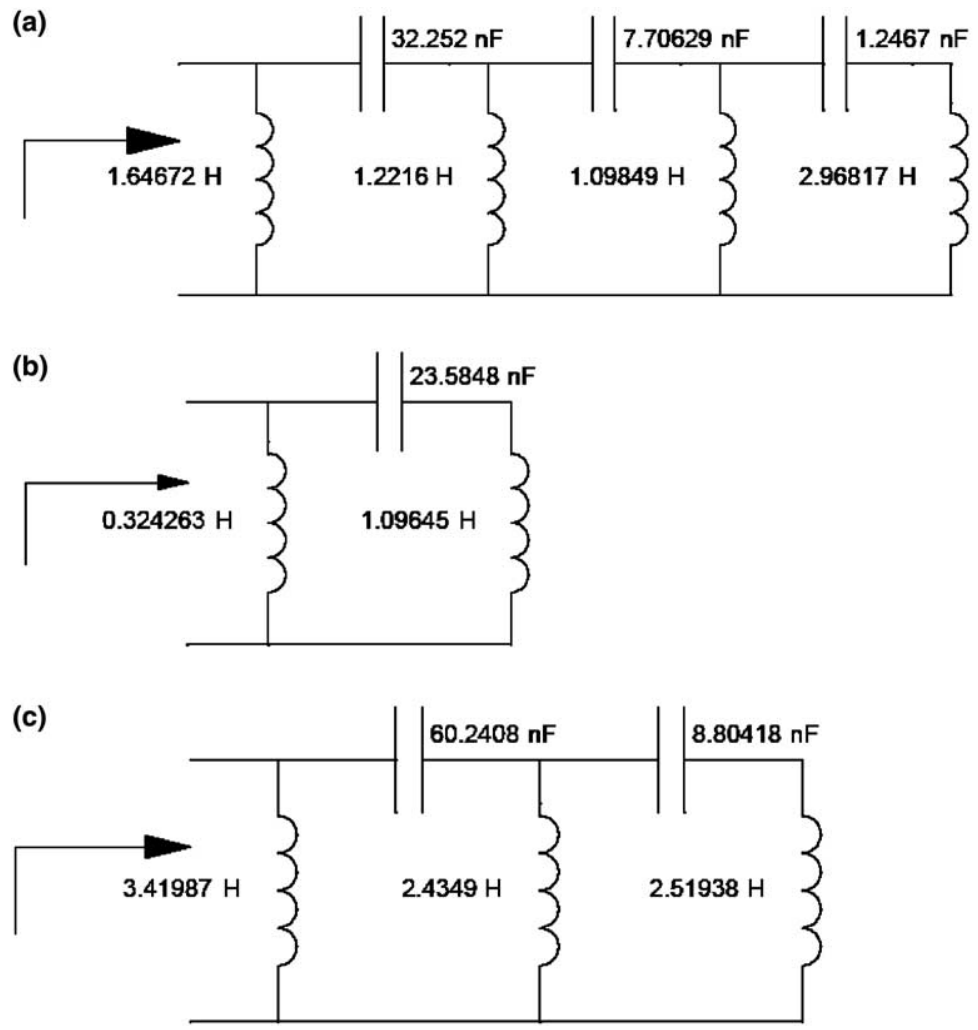

Fig. 11. For a cantilever aluminum beam, impedance for controlling (a) the first four vibration modes, (b) the first two vibration modes, and (c) the first three vibration modes.

which is the impedance of a capacitor (say $\left.C_{1}\right)$ of capacitance $1 / \bar{R}_{1}\left(\bar{R}_{1}\right.$ is the residue at $s=0$ of $Z_{1}(s)$ ) connected in series with an inductor of impedance $Z_{2}(s)$. This procedure can be iterated until $Z_{k}(s)$ or $Y_{k}(s)$ reduces to the impedance/admittance of a single inductor/capacitor. The main steps of this procedure are given in Fig. 10. The schematics of the LC TTNs which provide impedances needed in the applications 
presented in the previous sections are shown in Fig. 11a-c. A simple Mathematica code, which implements the Cauer method, has been developed and used to obtain these results.

\section{References}

Alves Rade, Domingos, Valder Jr., Steffen, 2000. Optimisation of dynamic vibration absorbers over a frequency band. Mech. Syst. Signal Process. 14, 679-690.

Andreaus, U., dell'Isola, F., Porfiri, M., 2004. Piezoelectric passive distributed controllers for beam flexural vibrations. J. Vib. Control $10,625-659$.

dell'Isola, F., Vidoli, S., 1998. Continuum modelling of piezo-electro-mechanical truss beams: an application to vibration damping. Arch. Appl. Mech.--Ing. Arch. 68, 1-19.

Fleming, A.J., Reza Moheimani, S.O., 2002. Optimization and implementation of multimode piezoelectric shunt damping systems. IEEE/ASME Trans. Mechatron. 7, 87-94.

Hagood, N.W., von Flotow, A., 1991. Damping of structural vibrations with piezoelectric materials and passive electrical networks. J. Sound Vib. 146, 243-268.

Hollkamp, J.J., 1994. Multimode passive vibration suppression with piezoelectric materials and resonant shunts. J. Int. Mater. Syst. Struct. 5, 49-57.

Lesieutre, G.A., 1998. Vibration damping and control using shunted piezoelectric materials. Shock Vib. Digest 30, $187-195$.

Park, C.H., Inman, D.J., 2003. Enhanced piezoelectric shunt design. Shock Vib. Digest 10, $127-133$.

Vidoli, S., dell'Isola, F., 2000. Modal coupling in one-dimensional electromechanical structured continua. Acta Mech. 141 (1-2), 3750 .

Vidoli, S., dell'Isola, F., 2001. Vibration control in plates by uniformly distributed actuators interconnected via electric networks. Eur. J. Mech. A/Solids 20, 435-456.

Walsh, J.L., 1960. Interpolation and Approximation by Rational Functions in the Complex Domain. American Mathematical Society. Wu, S.-Y., 1998. Method for multiple mode piezoelectric shunting with single PZT transducer for vibration control. J. Int. Mater. Syst. Struct. 9, 991-998. 